\title{
Le grand vénéréologue Philippe Ricord (1800-1889) et la distinction définitive entre la gonococcie et la syphilis
}

\author{
Lazaros VLADIMIROS, Aristea MISIAKOULI, Marianna KARAMANOU, \\ Georges ANDROUTSOS
}

Service d'Histoire de la Médecine, Faculté de Médecine, Université d'Athènes, Grèce

\section{RESUME}

Philippe Ricord, célèbre syphiligraphe français, a le mérite d'avoir fait le premier la distinction entre la syphilis de la blennorragie et d'avoir créé la fameuse école française de syphiligraphie.

Mots clés : Philippe Ricord, célèbre syphiligraphe français, syphilis, blennorragie

\section{GENERALITES}

La confusion la plus totale entre la syphilis et la gonorrhée a persisté pendant plusieurs siècles. Cette étrange confusion entre deux maladies pourtant si différentes provenait sans doute de leur coexistence fréquente durant les 7 derniers siècles où la nosologie avait progressé très lentement [1].

Le mérite d'avoir établi clairement que la syphilis et la gonorrhée sont deux maladies distinctes revient à Benjamin Bell (1749-1806) en 1793 [8] et à Joseph François Hernandez (1769-1835) en 1812 [10].

Malgré les expériences de Hernandez qui avait prouvé la non identité de la syphilis et de la blennorragie, il fallut attendre encore un tiers de siècle, pour que Philippe Ricord jugeât en dernier ressort. En 1843, Pierre Louis Alphée Cazenave
(1795-1877), médecin de Saint Louis, écrivait encore : " J'ai vu en un mot, après la blennorragie comme après le chancre, des syphilides graves. J'ai vu la blennorragie être suivie, comme le chancre, d'ulcérations des muqueuses, de caries des os, et même de cet état tout particulier qu'on appelle la cachexie syphilitique » [5]. Enfin, en 1838, venait Ricord, établissant, sans appel possible, que chancre et blennorragie représentent deux entités distinctes [15].

\section{VIE ET CARRIERE DE RICORD}

Ricord (Figure 1) naquit le 10 décembre 1800, à Philadelphie des États-Unis, de parents français - français lui-même, tout ce qu'il y a de plus français. Ricord commença ses études médicales en Amérique, chez un herboriste (pharmacien). Mais au bout d'un an, il jugea prudent pour ses ambitions d'aller se former à une meilleure école, et, au commencement de l'année 1820 , il se faisait inscrire à la Faculté de médecine de Paris. Huit mois plus tard, il concourait pour l'externat, un an après pour l'internat où il était admis le treizième de sa promotion [6]

Sa présence d'esprit dans les concours, l'acuité de son intelligence, la finesse de ses réparties, dont quelques unes sont restées légendaires et se répètent de générations en générations, le firent aussitôt remarquer. Son internat fut très brillant ; aussitôt sa thèse passée, il concourut pour une place de chirurgien des hôpitaux, et fut nommé d'emblée.

Mais une irrégularité commise à propos d'un autre candidat fit casser le concours, et Ricord se trouva sur le pavé de Paris,

\section{Correspondance :}

Pr Georges ANDROUTSOS - 1 rue Ipeirou, 10433, Athènes, Grèce - Email Iyon 48@otenet.gr 


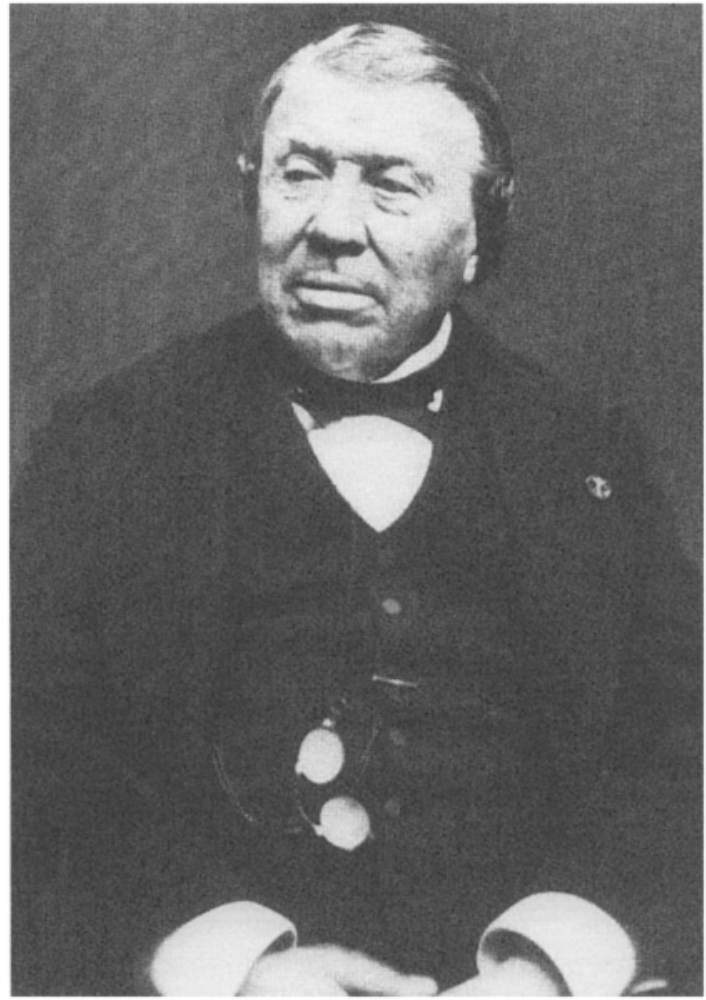

\section{Figure 1 : Philippe Ricord.}

ayant pour toute fortune, neuf francs en poche. II partit donc, pour revenir un jour en force, et il alla pratiquer la médecine à Croüy-sur-Ourq, dans un village où il n'avait pour rivaux que de modestes officiers de santé.

Ricord fit, d'abord, beaucoup de visites à dix sous, courut sous la pluie les chemins creux, montant un vieux cheval cosaque qu'il avait payé soixante francs ; peu à peu, il en vint à hausser le prix de ses honoraires, renonçant à la clientèle proprement dite, voyant surtout les malades des autres en consultations [3].

Si bien que, vingt mois après son départ de Paris, il y rentrait avec douze mille francs d'économies. Même dans ses plus durs moments, il n'avait pas oublié cette place de chirurgien des hôpitaux qu'il avait une fois gagnée et qu'il voulait reconquérir ; et dans ses tournées de médecin de village, il emportait ses notes et ses cahiers d'étude dans les fontes à pistolets de sa selle. Il concourut pour la seconde fois, et fut nommé en tête de la liste.

Le hasard le désigna pour l'hôpital du Midi. II y est resté jusqu'à sa retraite. Il devint professeur de Clinique médicale spéciale à partir de 1834. C'est là qu'il fit les découvertes qui ont illustré son nom ; là que dans l'inextricable confusion qui régnait avant lui, il sut faire la lumière, classer les faits, grouper les symptômes, enseigner aux médecins du monde entier les lois du diagnostic et la manière de traiter des maux vraiment terribles dans la première moitié du $19^{\mathrm{e}}$ siècle.

Vite célèbre pour ses cours brillants où affluent élèves et médecins français et étrangers, ainsi que pour la clientèle privée et huppée qui défile à son hôtel de la rue de Tournon, il est à la fois un mondain brillant et un savant authentique. II va rester près de trente ans à l'hôpital des Vénériens (qui prendra en 1836 le nom d'hôpital du Midi, lorsqu'un nouvel hôpital sera créé à Lourcine pour les femmes vénériennes) avant de se consacrer exclusivement à sa clientèle privée, de plus en plus nombreuse. Médecin de Napoléon III, comblé d'honneurs, il meurt en 1889, à 89 ans. En 1893, l'hôpital du Midi prendra le nom d'hôpital Ricord, lequel sera lui-même englobé dans l'hôpital Cochin en 1903 [14].

\section{RICORD : UNE PERSONNALITE HORS DU COMMUN}

Ricord a continué à exercer jusqu'à ses derniers jours ! En effet, pour quiconque le connaissait un peu, il est était tout à fait inadmissible qu'un pareil homme, en dépit de ses quatrevingt-neuf ans puisse se résigner au repos.

Non seulement il donnait chez lui chaque jour de nombreuses consultations, mais il allait voir des malades chez eux et retrouvait, pour opérer, la plus invraisemblable sûreté de main. Quelques jours avant sa mort, il alla en soirée. " Seulement, a-t-il coutume de dire, je ne peux plus faire de longues marches : aussi, me suis-je décidé à ne plus suivre d'enterrement, pas même le mien, puisque je tiendrai la tête du cortège ".

Qui ne connaissait son hôtel de la rue de Tournon; l'antichambre où, pendant trente ans, le même valet de chambre introduisait tant de milliers de patients ; le grand salon, ayant, à droite de la cheminée, le buste d'Asclépios, à gauche, le buste de Ricord - le dieu grec de la médecine faisant le pendant au demi-dieu de la rue de Tournon - et le cabinet du docteur avec sa vaste bibliothèque, et murs tapissés des innombrables portraits du maître ?...

C'est dans cette pièce qu'il recevait. Frileusement assis près de la cheminée, dans un fauteuil empire à cuivres qui tenait du trône et de la chaise curule, une main s'appuyant à la table, l'autre main frictionnant un genou, Ricord accueillait le visiteur avec le plus charmant sourire, en s'excusant de ne point se lever.

Puis il écoutait, les paupières mi-closes, les lèvres entr'ouvertes, les cheveux frisés et grisonnants sous la calotte de soie noire ; sa pâle et grasse figure glabre s'immobilisait inclinée en avant ; son triple menton rabelaisien s'écrasait un peu sur sa cravate jusqu'à venir toucher la rouge rosette de sa boutonnière ; et il avait l'air, ainsi, d'un vieux Silène aimable qui allait dormir.

Cependant, il ne perdait la moindre parole de ses patients, les interrogeait avec la plus parfaite lucidité; puis il parlait, évitant les gestes à ses membres rhumatisants, émaillant son discours de ses plus goguenardes plaisanteries. Enfin, il congédiait ses patients du même sourire inoubliable.

II faudrait un bon gros volume pour raconter avec détails une existence aussi bien remplie ! 


\section{RICORD : UN GÉANT DE LA SYPHILIGRAPHIE}

Ricord, lorsqu'il prit son poste en 1830 à l'hôpital du Midi (Vénériens), ne connaissait rien à la question de la syphilis [21]. Au travail, Ricord fait preuve d'une observation clinique rigoureuse, étant par exemple l'un des premiers à utiliser systématiquement le spéculum. Ricord avait su rejeter hors du cadre de la vérole la blennorragie, la balanoposthite, la vaginite, et les végétations, puisqu'il s'écriait : " II faut en finir avec les vieilles croyances. II faut briser cette unité factice des maladies vénériennes ou syphilitiques. Hors de la syphilis tout ce qui n'est pas la syphilis ! " Ce même Ricord n'en écrivait pas moins ailleurs : " Dans tous les cas (et ils sont déjà nombreux) où l'inoculation d'accidents secondaires ou tertiaires a pu être pratiquée sur des sujets sains, dans les conditions d'une expérimentation sérieuse, elle n'a donné lieu qu'à des résultats absolument négatifs. " II croyait également que la maladie rendait le sujet réfractaire à une nouvelle inoculation : "La syphilis ne se double pas. " [11].

Voilà comment Ricord, dans ses conclusions, sépare nettement blennorragie et syphilis : « Un contagium vénérien produit toujours des chancres quand on l'a prélevé sur un premier chancre. L'écoulement urétral inoculé sous la peau ne produit aucune lésion. II faut donc séparer la blennorragie du mal vénérien mais, néanmoins, elle est parfois inoculable, comme toute inflammation catarrhale, tel le coryza. " [15].

Ricord établit en outre des propositions révolutionnaires pour l'époque : la syphilis par bubon d'emblée n'existe pas ; seule, la syphilis est suivie d'accidents constitutionnels ; elle rend le sujet qui en est atteint réfractaire à une nouvelle inoculation (" la syphilis ne se double pas ") ; ses manifestations sont régies par une loi chronologique (" l'ordre et la discipline existent donc là où l'on s'obstinait à ne voir que confusion, dérèglement, hasard $») \ldots$

Voilà comment il expose ses idées révolutionnaires : «L'observation clinique m'a conduit à la classification suivante des symptômes de la syphilis : 1) Accident primitif, le chancre est dû à l'action directe du virus qu'il reproduit...2) Accidents consécutifs ou ceux qui arrivent de proche en proche, ou par simple extension du premier symptôme local, tels que de nouveaux chancres...3) Accidents secondaires ou d'infection générale...Ces phénomènes peuvent se transmettre par voie d'hérédité, et cela d'une manière incontestable, de la mère aux enfants... sans qu'ils aient des phénomènes primitifs... 4) Accidents tertiaires, arrivés à des époques indéterminées, mais le plus ordinairement longtemps après la cessation de l'accident primitif ; ne se montant sur le plus grand nombre des sujets, qu'alors que les symptômes secondaires ont déjà eu lieu, soit qu'ils aient disparu ou qu'ils persistent encore..., accidents qui ne s'inoculent plus. Parmi ces accidents tertiaires, il faut ranger les nodus, les tubercules profonds, ceux du tissu cellulaire, les périostoses, les exostoses, les caries, les nécroses, les tubercules syphilitiques du cerveau... " [15].

II tord aussi le cou aux doctrines des adeptes de Broussais : " Il s'agit maintenant de déterminer la source où se trouve la cause spécifique, le poison morbide qui produit la syphilis. Ce poison, on peut aujourd'hui l'appeler de son nom, c'est le virus syphilitique. Eh bien, ce virus, j'ai besoin de le rappeler, puisqu'on s'efforce de le faire oublier, ce virus était contesté et formellement nié quand j'entrepris mes premières recherches en syphiligraphie. C'était le temps où de nombreux médecins n'osaient plus lui donner ce nom sans crainte de se compromettre. C'était le temps où le savant Antoine Jourdan (1788-1848), dans un accès de bizarre colère, s'écriait : appelez-le comme vous voudrez, mais ne lui donnez pas le nom de virus ! " [16].

Ceux qu'il appelle plaisamment " les athlètes de l'école physiologiste » essaient de se défendre, lui déniant tout « rôle de réformateur et de chef de doctrine " et lui reprochant non seulement le fond mais aussi la forme, trop romantique, de ses ouvrages dont certaines expressions, pourtant, feront fortune, comme " les pléiades ganglionnaires " pour le rayonnement lymphatique des chancres indurés [7].

\section{LES ERREURS DE RICORD}

Toutefois, les failles ne manquent pas dans l'œuvre clinique de Ricord : il ne mentionne pas le stade d'incubation ; sa thérapeutique est un retour au mercure comme remède spécifique après condamnation des antiphlogistiques chers aux physiologistes ; il nie tout pouvoir contagieux aux accidents secondaires et notamment aux plaques muqueuses sous prétexte qu'il ne peut les inoculer. Pourtant, William Wallace (1791-1837), à la même époque, prouve leur contagiosité en inoculant, lui, des hommes sains ! Mais Ricord, à la tribune de l'Académie de médecine en 1852, maintient ses conclusions antérieures : " Je veux, dit-il, pour me faire changer d'opinion qu'on me donne des faits plus probants » [9]. Aussi, les travaux de son élève Joseph Rollet (1824-1894) qui émet des critiques prudentes sur la technique des inoculations de son maître, ne le feront pas changer d'avis.

C'est alors qu'à l'Antiquaille de Lyon, en 1856, Rollet, chirurgien-major de l'Hôpital, inocula la matière d'un chancre induré à divers syphilitiques, secondaires et tertiaires. Son entreprise n'aboutit pas, au lieu que le même geste, effectué sur un chancrelleux, provoqua, dix-huit jours après, l'apparition d'une papule. La démonstration était faite. Elle fut parfaite par Rollet, qui déduisit d'essais et d'observations ultérieurs, la notion d'une période d'incubation du chancre syphilitique, en moyenne 25 jours, par opposition à ce qui se passait pour l'ulcus molle, dont le temps de latence était des plus courts [12].

Contre ces acquisitions, Ricord s'inscrivit avec âpreté. «Un œil attentif et expérimenté " eût saisi, selon lui, le début instantané du chancre syphilitique. Ce que l'on dénommait une période d'incubation n'était qu'un temps d'" inobservation». Et continua, Ricord son offensive. "Que les amis du médecin de Lyon veuillent donc nous pardonner, si le chancre mulet, froidement examiné, va dans un instant disparaître avec la fumée de l'encens que ses jeunes admirateurs brûlent devant lui » [4].

Dans un débat devant l'Académie de médecine, Rollet s'opposa à Ricord à propos des contaminations lors de la vaccination antivariolique [18]. Ricord s'obstinait à faire état de ses expériences, aussi mal orientées que jadis celles de 
Hunter, et niait éperdument la contagiosité des accidents secondaires. Finalement, à partir de 1853 , Ricord comprendra que les lésions secondaires peuvent aussi être contagieuses.

De même, bien qu'il reconnaisse que le chancre induré est infectant et rend le sujet réfractaire à une réinoculation, et que le chancre mou est une lésion locale indéfiniment réinoculable au porteur, Ricord, après 1040 inoculations, avait conclut simplement à la diversité d'aspects ! Opérant sur le chancreux lui-même, il ne put se convaincre de la nature différente des deux accidents. II resta uniciste et il a écrit à ce propos : " Le chancre induré est indolent, suppure peu, s'accompagne de ganglions peu volumineux et sans réaction inflammatoire. Le chancre mou est ulcéreux et le pus qu'il sécrète en abondance est indéfiniment inoculable au porteur. Au premier succède fatalement l'infection constitutionnelle dont la guérison n'est obtenue que par le mercure, tandis que le second, accident purement local, n'exige pour se cicatriser que l'emploi de topiques » [17].

C'est Léon Bassereau (1810-1887) qui, en 1852, affirme le premier l'individualité du chancre mou (même lorsque ce dernier s'associe à la syphilis dans le chancre mixte), abattant ainsi le dernier pan de l'édifice uniciste [2].

\section{LES PROGRES DE LA VENEREOLOGIE APRES RICORD}

Après la flambée initiale du $16 e$ siècle, la recherche sur la syphilis présente une stagnation. La maladie ne recommence à susciter des travaux intéressants qu'au cours du $18^{\mathrm{e}}$ siècle avec des auteurs comme Hermann Boerhaave (1668-1738), puis Jean Astruc (1684-1766). En fait, c'est surtout au cours du $19^{\mathrm{e}}$ siècle que les avancées les plus spectaculaires sont réalisées.

Dès la fin du $18^{e}$ siècle avec John Hunter (1728-1793) et surtout au cours du $19 \mathrm{e}$ siècle avec Ricord, les auteurs ont appris à reconnaître deux types de chancres : le chancre induré dû à Treponema pallidum, l'agent de la syphilis, et le chancre mou (causé par Haemophilus ducreyi).

Le début du $20^{\mathrm{e}}$ siècle sera décisif dans la caractérisation définitive de l'agent responsable de cette maladie qui sévit depuis 4 siècles. Tout d'abord, Émile Roux (1853-1933) et Élie Metchnikoff (1845-1916), à l'institut Pasteur de Paris, réalisent la syphilis expérimentale chez les animaux entre 1903 et 1905 en inoculant et en décrivant la maladie chez les singes. Avant que Fritz Schaudinn (1871-1906) ne découvre l'agent responsable on disait ironiquement que " cent vingt-cinq causes pour la syphilis ont été établies au cours des vingtcinq dernières années ".

Schaudinn met en évidence, le 3 mars 1905, sur des étalements provenant d'une papule, de très fins spirochètes qu'il baptise spirochaeta pallidum, mais sans affirmer qu'il s'agit de l'agent responsable de la syphilis. Par la suite, une étude cytologique est entreprise par Schaudinn à partir d'échantillons sur des ganglions lymphatiques syphilitiques. Quelques spirochètes sont caractérisés et photographiés. Cette fois-ci, Schaudinn a la preuve formelle que ces spirochètes, qui prennent leur nom définitif de Treponema pallidum, sont bien la cause de la maladie. Aujourd'hui encore, il semble difficile de croire qu'en moins de 10 ans d'activité, Schaudinn ait pu élucider un si grand nombre d'énigmes biologiques majeures [20].

Une année seulement après la découverte de l'agent responsable, August von Wassermann (1866-1925) et son collègue Albert Neisser (1855-1916) annoncent à Berlin, le 10 mai 1906, la mise au point d'une méthode de sérologie pour le diagnostic de la syphilis [19].

Malgré ses nombreux défauts et bien qu'elle soit peu spécifique, la réaction de Bordet-Wassermann, dite BW, connaîtra alors un succès considérable pour le diagnostic de la syphilis. Le test sera amélioré plus tard, à la suite des travaux de Mary C. Pangborn (en 1941), qui permettront de standardiser la technique et de proposer un test plus sensible et spécifique [13]

\section{CONCLUSION}

Le nom de ce célèbre médecin symbolise à lui seul la syphiligraphie du milieu du 19 e siècle. Ses inventions en syphiligraphie sont capitales et lui valent un nom, plus que fameux : historique.

La renommée de Ricord fut promptement européenne. Très conscient de sa valeur - avec cet amour de la conquête et ce besoin d'emplir le monde qu'ont les hommes à qui tout réussit, et qui est bien plutôt une forme de l'optimisme qu'une manifestation d'orgueil vulgaire - Il a toujours soigné sa gloire très tendrement.

Homme d'esprit, parfois mordant mais médecin honnête il se refusera à pratiquer des inoculations sur des sujets sains comme le faisaient certains de ses contemporains. Ricord consacrera toute sa vie aux maladies vénériennes en cumulant richesse, honneurs et distinctions.

C'est sur le champ de bataille du Bourget qu'il a gagné sa croix de grand-officier de la Légion d'Honneur. Tout jeune, puisqu'il n'avait alors que soixante-dix ans, chef des ambulances du siège, il pansait fort tranquillement les blessés sous une grêle de balles, assisté seulement de son valet de chambre. Vint à passer un officier d'ordonnance, lequel cria au domestique : " Dites au docteur de s'en aller de là, s'il ne veut pas recevoir un mauvais coup ! » Ricord avait entendu et paisible, il répliqua, dans le tapage des mitrailleuses : « Répondez que je ne reçois pas ; je ne suis pas ici chez moi I ", puis se remit à la besogne. A lui seul, le mot vaut la décoration.

L'oeuvre œuvre de Ricord fut essentielle car, malgré les travaux d'Hernandez, c'est seulement avec lui que seront définitivement séparées syphilis et blennorragie. Il aura l'immense mérite de faire sortir les maladies vénériennes du chaos où elles étaient en 1830. Ricord a formé de nombreux élèves : trois d'entre eux comptent parmi les plus grands vénéréologues du 19 e siècle : Joseph Rollet, Alfred Fournier et Léon Bassereau. 


\section{REFERENCES}

1. ANDROUTSOS G., VLADIMIROS $L$ : De la gonorrhée à la blennorragie : Les grandes étapes historiques. Andrologie, 2007, 17 :141-153.

2. BASSEREAU L. :Affections de la peau symptomatique de la syphilis. Paris, Baillière, $1852: 36$.

3. BIANCHON H. : Grands Médecins d'aujourd'hui. Paris, Société d'éditions scientifiques, 1891 : 391-398.

4. BURGUIN R., LAUGIER P. : Histoire des maladies vénériennes. In: Histoire de la médecine de la pharmacie de l'art dentaire et de l'art vétérinaire. Paris, Editions Albin Michel/Laffont/Tchou, 1978, 8 vol., t. IV : 340-341.

5. CAZENAVE P. : Syphilides. Paris, Baillière, 1843.

6. COLIN A. : Philippe Ricord. In : Dictionnaire des noms illustres en médecine. Bruxelles, Prodim, 1994 :165-166.

7. CORBINA. : Peurs et terreurs face à la contagion. Sous la direction de J.P. Bardet, P. Bourdelais, P. Guillaume, F. Lebrun, Q. Quetel. Paris, Fayard, $1988: 328-348$.

8. DUPONT M. : Benjamin Bell. Dictionnaire historique des médecins dans et hors de la médecine. Paris, Larousse/Bordas, $1999: 64$.

9. HANSEN W., FRENEY J. : Des bactéries et des hommes. Histoire des grandes maladies infectieuses et de leurs diagnostics. Paris, Privat, 2002 : 93-96.

10. HERNANDEZ J.F. : Essai analytique sur la non-identité des virus gonorrhoïque et syphilitique. Toulon, Doin, 1812.

11. JAUSION H., MÉDIONI G. : Histoire du mal vénérien. In : Histoire générale de la médecine, de la pharmacie, de l'art dentaire et de l'art vétérinaire. Sous la direction de Laignel-Lavastine. Paris, Albin Michel, 1938, t. II : 465-566.

12. MORNEX R., DUCOURET B., FAURE O. : L'Antiquaille de Lyon. Histoire d'un hôpital. Lyon, Edition Lieux Dits, 2003 : 97.

13. PARIENTE Liliane : Petite Encyclopédie des maladies sexuellement transmissibles. Histoire et Actualité. Paris, Louis Pariente, 1993 : 253-255.

14. QUETEL C. : Le mal de Naples. Histoire de la syphilis. Paris, Seghers, $1986: 140-143$.

15. RICORD P. : Traité pratique des maladies vénériennes, ou Recherches critiques et expérimentales sur l'inoculation appliquée à l'étude de ces maladies. Paris, Doin, 1838.

16. RICORD $P$. : Lettres sur la syphilis, adressées à $M$. le Rédacteur en chef de l'Union médicale. Paris, $1851,12^{\mathrm{e}}$ lettre.

17. RICORD P. : Traité complet des maladies vénériennes. Clinique iconographique de l'hôpital des vénériens. Paris, Rouvier, 1851.

18. ROLLET J. : Traité des maladies Vénériennes. Lyon, P. Dorier, 1859.

19. RUFFIÉ J., SOURNIA J.C. : Les épidémies dans l'histoire de l'homme. Paris, Flammarion, 1984 : 186-188.

20. SALUZZO J.F. : Des hommes et des germes. Paris, Presses Universitaires de France, $2004: 77-79$.

21. TENON J. : Mémoires sur les hôpitaux de Paris. Paris, Imprimerie Pierre, 1788.

Manuscrit reçu : septembre 2008 ; accepté septembre 2008.

\section{ABSTRACT}

The great venereologist Philippe Ricord (1800-1889) and the final distinction between gonorrhoea and syphilis

\author{
Lazaros VLADIMIROS, Aristea MISIAKOULI, Marianna \\ KARAMANOU, Georges ANDROUTSOS
}

Philippe Ricord, a famous French venereologist, was the first to distinguish between syphilis and gonorrhoea and founded the famous French school of venereology.

Key words : Philippe Ricord, famous French venereologist, syphilis, gonorrhoea 